BMJ Open

Diabetes

Research

\& Care

\title{
Association of physiological and psychological health outcomes with physical activity and sedentary behavior in adults with type 2 diabetes
}

\author{
Jeanette M Garcia, ${ }^{1}$ Daniel Cox, ${ }^{2}$ David J Rice ${ }^{3}$
}

To cite: Garcia JM, Cox D, Rice DJ. Association of physiological and psychological health outcomes with physical activity and sedentary behavior in adults with type 2 diabetes. BMJ Open Diabetes Research and Care 2017:5: e000306. doi:10.1136/ bmjdrc-2016-000306

- Additional material is available. To view please visit the journal online (http://dx. doi.org/10.1136/bmjdrc2016-000306).

Received 28 July 2016 Revised 24 January 2017 Accepted 3 February 2017

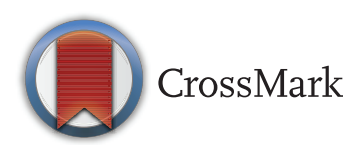

${ }^{1}$ Department of Education and Human Services, University of Central Florida, Orlando, Florida, USA ${ }^{2}$ Departments of Psychiatry, Internal Medicine, and Opthalmology, University of Virginia, Charlottesville, Virginia, USA

${ }^{3}$ School of Nursing and Health Sciences, Florida Southern College, Lakeland, Florida, USA,

Correspondence to Dr Jeanette M Garcia; Jeanette.garcia@ucf.edu

\section{ABSTRACT}

Purpose: To examine the association between change in moderate-to-vigorous physical activity (MVPA) and sedentary behavior (SB) over a 6-month period with physiological and psychological factors in adults with type 2 diabetes (T2D).

Methods: Participants included 26 middle-aged (mean age $=56.1 \pm 10.8$ years; $42 \%$ women), overweight/obese (mean body mass index (BMI) $=37.22 \pm 8.78 \mathrm{~kg} / \mathrm{m}^{2}$ ) adults who had been diagnosed with T2D within the past 5 years (mean $\mathrm{HbA} 1 \mathrm{c}=7.81 \%$ ). Participants underwent a physical examination, blood tests, and psychological questionnaires, including a self-report questionnaire that assessed the consumption of high glycemic and low glycemic load foods. Participants wore an Actigraph accelerometer for 7 days to assess MVPA and SB. All measures were collected at baseline and at the 6-month follow-up. Spearman rank correlations and regression models were conducted to examine the relationship between activity variables, and the association of activity measures with health outcomes at the 6-month follow-up.

Results: Decreases in duration of SB bouts and increases in MVPA were associated with decreased levels of $\mathrm{HbA1c}(p<0.05)$. Over $50 \%$ of the variance in $\mathrm{HbA1c}$ levels could be attributed to changes in MVPA and SB.

Conclusions: MVPA and SB were independently associated with diabetes-related health outcomes. Results suggest that emphasis should be placed on increasing MVPA while decreasing SB, particularly duration of SB bouts. This suggests that even small changes in daily behavior may contribute to improvement in diabetes-related health outcomes.

\section{INTRODUCTION}

There has been a rise in the prevalence of type 2 diabetes (T2D) in the USA, which may be correlated with the increasing levels of obesity, poor dietary habits, and physical inactivity. ${ }^{1}$ In fact, an estimated 29.1 million Americans are currently living with T2D, with 1.4 million new cases being diagnosed each year. ${ }^{2}$ Over 422 million cases of T2D have been diagnosed worldwide. ${ }^{1}$ Diabetes is

\section{Key messages}

- Physical activity and sedentary behavior are distinct behaviors associated with different diabetes-related outcomes.

- Decreases in uninterrupted bouts of sedentary behavior are associated with reduction in $\mathrm{HbA1C}$ levels.

- Increased moderate-to-vigorous physical activity is associated with a reduction in HbA1c levels and improvements in psychosocial outcomes such as a reduction in depressive symptoms.

- Small changes in sedentary behavior, such as breaking up uninterrupted bouts of sedentary behavior with a few minutes of light activity, may be significant enough to improve diabetes-related outcomes.

reported to be the 7th leading cause of death in the USA, and if left untreated, can lead to numerous health complications such as blindness, neuropathy, and infection. Through a combination of medication and healthy lifestyle behaviors (diet, physical activity), however, T2D can be effectively managed and individuals can live a full and healthy life. ${ }^{2}$

In response to the increase in prevalence of T2D, public health recommendations have promoted increases in moderate-tovigorous physical activity (MVPA). ${ }^{3}$ However, there is emerging evidence that attention should also be focused on sedentary behavior $(\mathrm{SB})$, which is directly related to chronic disease, independent of physical activity. ${ }^{4}{ }^{5}$ In fact, SB has been associated with obesity, abnormal glucose metabolism, and the metabolic syndrome. ${ }^{6}$ In addition, a recent meta-analysis by Biswas et $a l^{7}$ found a $34 \%$ higher risk for all-cause mortality for prolonged sedentary time, even after adjusting for MVPA. Another meta-analysis by Wilmot et $a l^{8}$ found similar results, reporting that greater levels of $\mathrm{SB}$ were associated with a twofold increase in relative risk of T2D 
$(\mathrm{RR}=2.12(1.61-2.78))$ as compared to the group with the lowest levels of SB.

In addition to the effects of total sedentary time, the manner in which it is accumulated may also be important. Single bouts of prolonged, uninterrupted SB has been shown to decrease insulin sensitivity in adults, while additional evidence shows that breaking up bouts of SB may lead to improved metabolic profiles, however, few studies have examined these associations in individuals with T2D. ${ }^{39}$

In addition to physiological outcomes, evidence suggests that psychological outcomes may be connected to activity behavior, such that increases in MVPA may be associated with improved mood and psychological well-being in adults. ${ }^{10}$ Although the literature on SB and psychological well-being is less known, there have been a few studies suggesting that increases in SB may be associated with adverse behavioral outcomes such as depression. ${ }^{11}$ Poorer psychological health may be associated with poorer prognosis in individuals with T2D. ${ }^{12}$ Therefore, the purpose of this study was to examine the association between changes/improvements in MVPA and SB over a period of 6 months with physiological and psychological health outcomes for individuals recently diagnosed with T2D.

\section{METHODS}

Participants were 26 adults, ranging in age from 33 to 77 years old, with T2D (mean age: $56.1 \pm 10.8$ years, $42 \%$ women, mean of $2.1 \pm 1.7$ years since diagnosis) who were part of a larger study ${ }^{13}$ designed to examine the effects of a lifestyle intervention on diabetes outcomes. Out of the 26 adults, 14 participants were part of the intervention arm, which consisted of an intensive lifestyle modification group that emphasized better nutritional choices and increased exercise, and the remaining 12 were part of the control group, which focused on diabetes support and education. These 26 participants were included in this smaller analysis because they had complete objective measures of physical activity and SB at baseline and postintervention. Additional inclusion criteria of the parent study were: have been diagnosed with T2D within the past 5 years, between 24 and 80 years of age, have an Hbalc level of $>7.0 \%$, and the approval of their treating physician to participate in the study and signed an informed consent approved by the University of Virginia Institutional Review Board.

Participants completed a physical examination, blood tests, a 6 min walk test to assess physical fitness, psychological questionnaires, and wore devices to assess their MVPA and SB for 1 week at baseline and at 6-month follow-up. Details of these measures are presented below.

\section{Physiological measures}

All physiological measures were assessed during a clinic visit at baseline and then at the 6-month follow-up visit. Height was measured to the nearest millimeter using a wall-mounted stadiometer. Weight was assessed to the nearest $0.1 \mathrm{~kg}$ using a spring scale that was calibrated daily. Metabolic markers were assessed using venous blood samples, following an overnight fast. Blood tests included: HbA1c TOSOH G7 (HPLC), CRP-HS Abbott Architect (latex immunoassay), and Insulin Immulite 2000 (sandwich immunoassay).

\section{Physical activity/sedentary behavior}

The ActiGraph GT3X+ device was used to assess physical activity (ActiGraph, LLC, Pensacola, Florida, USA). The ActiGraph has the ability to detect normal human motion while filtering out high-frequency vibrations that would artificially increase movement data, and has been validated for use in adults in laboratory and field studies. ${ }^{14}$ Data were collected in 1 min epochs and postprocessed, using ActiLife software, into counts per minute (CPM). Participants were instructed to wear the devices for 7 days, and to only remove them when they were sleeping or engaging in water-based activities (eg, swimming). To be included in the analysis, participants were required to have at least 3 days of at least $600 \mathrm{~min}$ of data per day, with at least two weekdays and one weekend. Times of $\geq 60 \mathrm{~min}$ of accelerometer CPM values $=0$ were considered times when the device was not worn and were excluded from the analysis. ${ }^{15}$ Values $\geq 2296$ CPM were classified as moderate-to-vigorous physical activity (MVPA) and values $<100$ CPM were classified as $\mathrm{SB}{ }^{14}{ }^{16}$ Bouts of SB were defined as average consecutive minutes spent in sedentary time, and breaks in sedentary time were defined as interruptions (>100 CPM) in sedentary bouts. Average steps per day were also recorded in participants.

\section{Aerobic fitness}

Participants were instructed to complete a 6 min walk test to assess exercise tolerance. ${ }^{17}$ This test is self-paced, and measures the distance an individual is able to walk over a total of $6 \mathrm{~min}$ on a hard, flat surface. The primary outcome is the total distance covered in meters that participants traveled over the $6 \mathrm{~min}$ time period. A lower score, which would reflect less distance covered in $6 \mathrm{~min}$, would indicate lower aerobic fitness. This instrument has high test-retest reliability, with an intraclass correlation (ICC) of 0.80 reported in a group of older adults who had chronic heart failure and associated comorbidities including diabetes and hypertension. ${ }^{18}$ Moderate to high relationships have been reported $(\mathrm{r}=0.56-0.88)$ between the 6 min walk test and peak oxygen uptake, assessed by maximal exercise testing, in older populations. ${ }^{19} 20$

\section{Psychosocial questionnaires \\ Quality of life}

Quality of life was assessed with the 26 item WHO Quality of Life (WHOQOL-BREF) Questionnaire. ${ }^{21}$ This questionnaire is a modified version of the original 100 item WHO Quality of Life (WHOQOL-100) Questionnaire, and consists of four of the following 
domains: physical health (seven items), psychological health (six items), social relationships (three items), and environmental health (eight items). The response items on the questionnaire ranged from 1 to 5 . Similar to WHOQOL-100, domain scores are calculated by multiplying the mean of all items in the domain by 4 . Previous studies have shown high correlations between the WHOQOL-BREF and the original WHOQOL-100, ranging from 0.89 (domain 3) to 0.95 (domain 1). Internal consistency was good, with Cronbach's alphas ranging from 0.66 (domain 3 ) to 0.84 (domain 1) in the current investigation.

\section{Empowerment}

Diabetes empowerment was assessed using the 8-item short form of the original 28-item Diabetes Empowerment Scale (DES-SF). ${ }^{22-23}$ The original questionnaire consisted of eight conceptual dimensions (assessing the need for change, developing a plan, overcoming barriers, asking for support, supporting oneself, coping with emotion, motivating oneself, and making diabetes care choices appropriate for one's priorities and circumstances). The 8 -item version was created by choosing from the 28 items that had the highest item to subscale correlation from each of the eight conceptual dimensions. Response options ranged from 1 (strongly disagree) to 5 (strongly agree). The questionnaire starts with the phrase 'In general, I believe that I...' and is followed by eight statements such as '...know what part(s) of taking care of my diabetes that I am dissatisfied with' and '... am able to turn $\mathrm{m}$ diabetes goals into a workable plan'. The DES-SF demonstrated good reliability with $\alpha=0.84$.

\section{Depressive symptoms}

Depressive symptoms were assessed using a shorter version of the Patient Health Questionnaire (PHQ). ${ }^{24}$ The PHQ-9 is the 9-item depression module from the original PHQ. This questionnaire can be used as a screening diagnostic tool for depressive symptoms, and to assess depression severity. The questionnaire opens with, 'Over the last 2 weeks, how often have you been bothered by any of the following problems?' and then lists nine statements that assess depressive symptoms such as 'little interest or pleasure in doing things' and 'feeling down, depressed, or hopeless'. The PHQ-9 score can range from 0 to 27 , since each of the 9 items can be scored as 0 (not at all) to 3 (nearly every day). Previous studies have reported excellent internal reliability, citing Cronbach's alphas of 0.86 and $0.89 .{ }^{25}$

\section{Diabetes-related stress}

Diabetes-related stress was assessed using the 5-item Problem Areas in Diabetes (PAID) Scale. ${ }^{26}$ This scale was created as a short-form version of the original 20-item PAID Questionnaire. The PAID-5 has a 5-point response option ranging from 0 (not a problem) to 4 (serious problem). Total scores on the PAID-5 can range from 0 to 20, with higher scores suggesting greater diabetes-related emotional distress. McGuire et $a l^{27}$ found that the PAID-5 had satisfactory sensitivity (94\%) and specificity $(89 \%)$ for recognition of diabetes-related emotional distress. Additionally, this measure reported good internal reliability, with Cronbach's alphas ranging from 0.83 to $0.86 .^{27}$

\section{Statistical analysis}

Descriptive statistics were calculated to determine how many of the participants met the PA recommendations of $30 \mathrm{~min}$ of daily MVPA, recommended by the American Heart Association, at baseline and 6 months. Physiological and psychological health factors were assessed by repeated measures t-tests to examine the change in values over the 6-month period. Additionally, repeated measures t-tests were also used to examine the differences between activity measures at baseline and at the 6-month follow-up. Spearman Rank correlations were conducted to examine the association among activity variables. Linear regression models, controlling for age, gender, body mass index (BMI), were conducted to examine whether activity variables (MVPA, SB, and fitness) independently predicted diabetes-related outcomes. All analyses were conducted in SAS V.9.3 with a significance level set $\alpha<0.05$.

\section{RESULTS}

On average, participants were obese, engaged in low levels of MVPA, and were largely sedentary during the day. Approximately $50 \%$ of participants were on diabetes-related medications. At baseline, only two out of the $26(8 \%)$ met the recommended daily MVPA levels of $30 \mathrm{~min}$ per day, and three (12\%) met the recommended MVPA levels at 6 months. Over the 6-month period, participants increased their MVPA, on average, by 6 min per day, while decreasing their total time in SB by around $20 \mathrm{~min}$. In addition, participants decreased their uninterrupted bouts of SB by almost $10 \mathrm{~min}$. While trends existed for increases in average steps per day ( 5601.73 vs $6603.95, \mathrm{p}=0.07$ ) and greater MVPA levels at 6 months (20.8 vs 27.04 MVPA min/day, $\mathrm{p}=0.06$ ), no significant differences existed for aerobic fitness or any of the SBs.

Participant physiological and psychological health factors at baseline and 6 months are displayed in table 1 . HbAlc levels significantly decreased at 6 months compared with baseline levels (7.52 vs 8.01, $\mathrm{p}=0.01)$. Additionally, feelings of empowerment over diabetes significantly improved at 6 months (33.15 vs 26.5, $\mathrm{p}<0.0001)$. No significant changes in any of the other physiological or psychological variables occurred over the 6 months.

\section{Relationship among activity and sedentary variables}

Correlations among changes in activity and sedentary variables are displayed in table 2. Spearman correlations 
indicated that there were significant correlations in activity variables with change in average steps per day associated with a change in MVPA $(\mathrm{r}=0.85, \mathrm{p}<0.0001)$ and a trend toward an association with change in aerobic fitness $(r=0.34, p<0.1)$. Increases in SB per day was associated with increases in uninterrupted bouts of SB $(\mathrm{r}=0.44, \mathrm{p}<0.05)$. Inverse correlations existed between activity and sedentary variables with decreases in MVPA associated with increases in SB $(r=-0.48, \mathrm{p}<0.05)$ and increases in bouts of SB $(r=-0.78, \mathrm{p}<0.0001)$. Finally, a decrease in average steps per day was associated with

Table 1 Baseline and 6 month physiological and psychological health variables

\begin{tabular}{|c|c|c|}
\hline Variables & Baseline & $\begin{array}{l}\text { 6-month } \\
\text { follow-up }\end{array}$ \\
\hline $\mathrm{BMI}$ & $37.61(8.84)$ & $36.31(9.4)$ \\
\hline Insulin & $17.52(11.73)$ & $17.84(17.54)$ \\
\hline $\mathrm{HbA1c}$ & $8.1(0.83)$ & $7.52(1.26)^{\star \star}$ \\
\hline CRP & $7.36(7.4)$ & $6.08(6.3)$ \\
\hline Systolic BP & 128.63 (17.97) & 127.67 (15.12) \\
\hline Quality of life & $50.67(6.86)$ & $52.67(6.56)$ \\
\hline Depression & 3.33 (3.05) & $2.89(3.14)$ \\
\hline Diabetes concern & $7.21(4.83)$ & $8.26(6.86)$ \\
\hline Diabetes empowerment & $26.5(9.44)$ & $33.15(5.46)^{\star \star}$ \\
\hline
\end{tabular}

increases in $\mathrm{SB} \quad(\mathrm{r}=-0.42, \mathrm{p}<0.05)$ and bouts of $\mathrm{SB}$ $(\mathrm{r}=-0.65, \mathrm{p}<0.01)$.

\section{Regression results for activity variables}

Regression models indicated that increases in MVPA $(\beta=$ $-0.04, \mathrm{SE}=.01, \mathrm{p}<0.01)$ and average steps walked per day $(\beta=-0.003, \mathrm{SE}=0.00, \mathrm{p}<0.01)$ were associated with lower HbA1c levels at the 6-month follow-up. Table 3 displays the regression results for the activity behaviors. For the psychological variables, an increase in MVPA was associated with decreases in depression $(\beta=-0.07, \mathrm{SE}=0.03$, $\mathrm{p}<0.05)$ and self-reported concerns about diabetes $(\beta=$ $-0.16, \mathrm{SE}=0.07, \mathrm{p}<0.05)$. Finally, an increase in aerobic fitness was associated with an increase in overall quality of life $(\beta=0.01, \mathrm{SE}=.01, \mathrm{p}<0.05)$.

\section{Regression results for SBS}

Regression models indicated that reductions in longer bouts of uninterrupted SB was associated with reduced HbA1c levels at the 6-month follow-up $(\beta=0.17, \mathrm{SE}=.08$, $\mathrm{p}<0.05)$. Results for SBs are displayed in table 4 . Increased breaks in SB was associated with increased insulin levels $(\beta=0.44, \mathrm{SE}=.16, \mathrm{p}<0.05)$. Finally, although not significant, there was a trend toward longer bouts of uninterrupted SB being associated with greater concern of diabetes $(\beta=0.68, \mathrm{SE}=0.47, \mathrm{p}<0.1)$.

Since bouts of SB and the activity variables were associated with HbA1c levels, bouts and MVPA were entered

Table 2 Relationship among change in activity variables

\begin{tabular}{|c|c|c|c|c|c|c|}
\hline Variables & $\Delta$ MVPA & $\Delta$ Steps & $\Delta$ Fitness & $\Delta \mathrm{SB}$ & $\Delta$ SB bouts & $\Delta$ SB breaks \\
\hline$\triangle \mathrm{MVPA}$ & - & $0.85^{\star \star \star}$ & 0.16 & $-0.48^{*}$ & $-0.78^{\star \star \star}$ & -0.1 \\
\hline$\Delta$ Steps & $0.85^{\star \star \star}$ & - & 0.34 & $-0.42^{*}$ & $-0.65^{\star \star \star}$ & -0.12 \\
\hline$\Delta$ Fitness & 0.16 & $0.34 †$ & - & 0.12 & -0.07 & 0.03 \\
\hline$\Delta \mathrm{SB}$ & $-0.48^{*}$ & $-0.42^{*}$ & 0.12 & - & $0.44^{*}$ & -0.22 \\
\hline$\Delta \mathrm{SB}$ bouts & $-0.78^{\star \star \star}$ & $-0.65^{\star \star \star}$ & -0.07 & $0.44^{*}$ & - & 0.2 \\
\hline$\Delta \mathrm{SB}$ breaks & -0.1 & -0.12 & 0.03 & -0.22 & 0.2 & - \\
\hline
\end{tabular}

${ }^{*} \mathrm{p}<0.05 ;{ }^{* * *} \mathrm{p}<0.001 ; \mathrm{tp}<0.01$.

MVPA, moderate-to-vigorous physical activity; SB, sedentary behavior.

Table 3 Regression results for activity behaviors

\begin{tabular}{|c|c|c|c|}
\hline Variables & $\Delta$ MVPA & $\Delta$ Total steps & $\Delta$ Fitness \\
\hline \multicolumn{4}{|l|}{ Physiological } \\
\hline BMI & $0.01(0.11)$ & $-0.00(0.00)$ & $-0.01(0.01)$ \\
\hline Insulin & $-0.04(0.21)$ & $-0.00(0.00)$ & $0.01(0.01)$ \\
\hline Hba1c & $-0.04(0.01)^{\star \star}$ & $-0.003(0.00)^{\star \star}$ & $-0.00(0.02)$ \\
\hline CRP & $-0.02(0.07)$ & $-0.00(0.00)$ & $-0.01(0.00)$ \\
\hline Systolic BP & $0.04(0.18)$ & $0.00(0.00)$ & $-0.01(0.01)$ \\
\hline \multicolumn{4}{|l|}{ Psychological } \\
\hline Quality of life & $0.05(0.08)$ & $0.0005(0.00)$ & $0.01(0.005)^{\star}$ \\
\hline Depression & $-0.07(0.03)^{\star}$ & $-0.00(0.00)$ & $-0.00(0.00)$ \\
\hline Diabetes concern & $-0.16(0.07)^{\star}$ & $-0.01(0.00)^{\star}$ & $-0.00(0.01)$ \\
\hline Diabetes empowerment & $-0.02(0.06)$ & $0.00(0.00)$ & $-0.00(0.00)$ \\
\hline
\end{tabular}


Table 4 Regression results for sedentary behaviors

\begin{tabular}{lcrr}
\hline Variables & $\Delta$ Total sedentary time & $\Delta$ Duration of bouts & $\Delta$ Number of breaks \\
\hline Physiological & & & $0.06(0.08)$ \\
BMI & $-0.04(0.03)$ & $-0.63(0.63)$ & $0.44(0.16)^{\star}$ \\
Insulin & $-0.05(0.1)$ & $0.94(1.59)$ & $0.02(0.01)$ \\
HbA1c & $0.004(0.00)$ & $0.017(0.08)^{*}$ & $0.04(0.05)$ \\
CRP & $-0.02(0.02)$ & $-0.1(0.42)$ & $0.07(0.15)$ \\
Systolic BP & $-0.02(0.06)$ & $-0.32(1.23)$ & $-0.04(0.06)$ \\
Psychological & & & $0.03(0.03)$ \\
Quality of life & $-0.01(0.03)$ & $0.12(0.5)$ & $0.07(0.06)$ \\
Depression & $0.01(0.01)$ & $0.17(0.28)$ & $-0.05(0.05)$ \\
Diabetes concern & $0.07(0.06)$ & $0.68(0.47) \dagger$ & $-0.05(0.18)$ \\
Diabetes empowerment & $-0.01(0.02)$ & $0.00(0.00)$ & $-0.03(0.16)$ \\
LGL & $-0.06(0.08)$ & $-1.5(1.39)$ & $0.63(1.29)$ \\
HGL & $0.05(0.06)$ & & \\
\hline${ }^{*}$ p $<0.05$. & & &
\end{tabular}

simultaneously into a multiple regression model, along with BMI (no other demographic variable was correlated with HbA1c). Increased MVPA $(\beta=-0.04, \quad \mathrm{SE}=0.01$, $p=0.01)$ and decreased bouts of $\mathrm{SB}(\beta=0.05, \mathrm{SE}=.02$, $\mathrm{p}=0.05$ ) were both significantly associated with decreased HbA1c levels, while BMI was not quite significant, although a trend remained $(\beta=0.05, \mathrm{SE}=0.03, \mathrm{p}=0.06)$. The overall model was significant $(\mathrm{p}=0.03)$, and the addition of these variables accounted for nearly $50 \%$ of the variance in HbA1c levels.

\section{DISCUSSION}

Similar to previous studies, MVPA and SB were only moderately correlated with each other, indicating that MVPA and SB are distinctly different behaviors. ${ }^{4}{ }^{72}$ In addition, participation in MVPA does not fully protect individuals from adverse health effects from high levels of $\mathrm{SB}$ as evidenced by the independent effect of SB and duration of sedentary bouts on HbAlc in the regression model. Despite the small sample size, the increased duration of uninterrupted bouts of SB and decreased activity levels were associated with increased $\mathrm{HbAlc}$ levels after 6 months, accounting for $50 \%$ of the variance in the model. This finding is informative to future research given that HbAlc levels are used to diagnose T2D and evaluate the success of its treatment. These results that suggest HbAlc levels are influenced by MVPA have been supported by previous findings. ${ }^{29-31}$ Church $e t a l^{29}$ reported the effects of a 9 month aerobic and resistance training trial on 262 sedentary individuals with T2D, finding that the exercise training group experienced a significant reduction in HbAlc levels compared with the control group. Fewer studies have examined objective SB and HbAlc levels; however, studies examining insulin resistance and SB have found mixed results. ${ }^{30-31}$ Helmerhorst $e t a l^{30}$ found that time spent in SB was associated with fasting insulin; however, another study by Ekelund $e t a l^{11}$ found that MVPA, but not sedentary time, was associated with increased insulin resistance. With the significant decrease in HbAlc levels over the 6-month period in the current study, nine of the $26(35 \%)$ participants achieved HbA1c levels <7.0. This suggests that developing interventions that focus on increasing MVPA and breaking up long bouts of uninterrupted SB may help to control T2D and improve their metabolic health.

Interestingly, SB was not associated with any of the psychological health outcomes, while MVPA and average steps per day were associated with decreased levels of depression and concern over diabetes. This finding is supported by previous research that found increases in activity were associated with improved mood and decreased depression. ${ }^{10}{ }^{32}$ Previous literature has suggested that intensity is also associated with decreased depression which may explain why actively engaging in activity, but not SB, was associated with improved mood. ${ }^{32-34}$ Additionally, it could be speculated that participants felt better about the prognosis of their T2D, since they were actively engaging in behaviors that are known to improve their health such as increased MVPA. Since SB tends to be more of a passive behavior, participants may have been more aware of behaviors that increased MVPA, rather than behaviors that decreased sedentary time. Additional studies to compare the effects of PA and SB on psychological health are warranted. It would be of interest for future studies to actively track SB, and provide participants feedback for decreasing prolonged uninterrupted sedentary time and/or overall SB throughout the day. Similar to evidence suggesting the use of activity trackers to promote PA behavior through feedback, feedback on SB may allow participants to make even larger changes to decrease their SB.

Results of this study indicate that MVPA and SB are two distinct behaviors that are associated with different 
health outcomes status. These findings suggest that special emphasis should be placed on decreasing SB and breaking up bouts of SB during the day to improve diabetes-related physiological and psychological outcomes. This particular sample was obese, engaged in a low amount of MVPA, and accumulated nearly 10 hours of SB with the average duration of a bout of SB lasting $90 \mathrm{~min}$. However, small changes in activity behavior such as decreasing the duration of uninterrupted SB, was associated with significant improvements in $\mathrm{HbAlc}$ levels. For individuals who have not been active or suffer from chronic health problems, undertaking an exercise program may not be feasible, however, by just decreasing the amount of time sitting on a couch, individuals with T2D may experience positive health benefits such as a decrease in HbA1c levels.

\section{IMIPLICATIONS}

Results from this study carry several implications regarding future lifestyle interventions for obese individuals with T2D. This study found that SB and physical activity largely affect HbAlc levels, suggesting that to improve physiological profiles in individuals with T2D, interventions must focus on increasing MVPA and decreasing SB and prolonged bouts of SB behavior. Although much emphasis has been placed on increasing MVPA in these populations, alterations in SB patterns may lead to physiological improvements in several diabetes-related health outcomes as well. Future studies should further examine whether educating individuals with T2D on the importance of reducing SB to help improve their health could potentially lead to improvements in psychological outcomes, similar to MVPA. The significant relationships between lower HbAlc levels and physical activity, despite only modest increases in MVPA and decreases in sedentary bouts, suggest that even just small increases in activity and decreases in SB could bring about significant positive health improvements. These changes could include taking a 5 min walk during an 8 hour desk office job, or even breaking up long bouts of SB by standing and stretching every $30 \mathrm{~min}$. Additionally, the use of commercial pedometers that alert wearers when there has been no movement in the past 60 min may be beneficial in reminding individuals to break up SB bouts. Such small changes may be particularly appealing to an obese, sedentary population as an initial behavioral strategy.

Acknowledgements The study authors would like to thank the research staff who assisted with the study, and the participants who made this study possible. The authors also would like to acknowledge John R. Sirard for the use of his accelerometers which were used to assess activity behavior.

Contributors DC was the principle investigator on the project, helped to conceptualize the design of the project, supervised the data collection, and helped to prepare and revise the final manuscript. JMG helped to conceptualize the design of the project, performed the data analysis, was involved in the interpretation of data, helped to prepare and revise the final document, and, as the corresponding author, oversaw the contributions of all of the authors. DJR assisted with the data analysis and interpretation of the data, helped to prepare and revise the document, and was responsible for overseeing the revisions of the final document. All of the authors agree that they are accountable for the accuracy and integrity of all parts of the project.

Funding JMG was supported by the Cardiovascular Epidemiology Training Grant T32HL098048 in Behavior, the Environment, and Global Health from the National Heart, Lung, and Blood Institute, National Institutes of Health. The other authors received no specific grant from any funding agency in public, commercial, or not-for-profit sectors.

\section{Competing interests None declared.}

Ethics approval The study received ethics approval by University of Virginia Institutional Review Board.

Provenance and peer review Not commissioned; externally peer reviewed.

Data sharing statement Ownership of the data from the project will be shared between the three authors. Storage and security of the data will be the responsibility of the corresponding author, JMG, and the data will be housed on a dedicated hard drive using password-protected program. Any requests for access to the data will be reviewed by all three authors and the release of any portion of the data must be agreed on by all three of the authors.

Open Access This is an Open Access article distributed in accordance with the Creative Commons Attribution Non Commercial (CC BY-NC 4.0) license, which permits others to distribute, remix, adapt, build upon this work noncommercially, and license their derivative works on different terms, provided the original work is properly cited and the use is non-commercial. See: http:// creativecommons.org/licenses/by-nc/4.0/

\section{REFERENCES}

1. Global Report on Diabetes. Geneva: World Health Organization, 2016.

2. American Diabetes Association website. http://www.diabetes.org/ diabetes-basics/statistics/?loc=superfooter (accessed Jul 2016).

3. Pate RR, Pratt M, Blair SN, et al. Physical activity and public health: a recommendation from the procedure Centers for Disease Control and Prevention and the American College of Sports Medicine. JAMA 1995:273:402-7.

4. Chastin SFM, Palarea-Albaladejo J, Dontje ML, et al. Combined effects of time spent in physical activity, sedentary behaviors, and sleep on obesity and cardio-metabolic health markers: a novel compositional data analysis approach. PLOS ONE 2015;10: e0139984.

5. Larsen BA, Martin L, Strong DR. Sedentary behavior and prevalent diabetes in Non-Latino Whites, Non-Latino Blacks, and Latinos: findings from The National Health Interview Survey. J Public Health (Oxf) 2015;37:634-40.

6. Owen N, Healy GN, Matthews CE, et al. Too much sitting: the population-health science of sedentary behavior. Exerc Sport Sci Rev 2010;38:105-13.

7. Biswas A, Oh PI, Faulkner GE, et al. Sedentary time and its association with risk for disease incidence, mortality, and hospitalization in adults: a systematic review and meta-analysis. Ann Intern Med 2015;162:123-34.

8. Wilmot EG, Edwardson CL, Achana FA, et al. Sedentary time in adults and the association with diabetes, cardiovascular disease and death: systematic review and meta-analysis. Diabetologia 2012;55:2895-905.

9. Healy GN, Dunstan DW, Salmon J, et al. Breaks in sedentary time. Diabetes Care. 2008;31:661-6.

10. Penedo FJ, Dahn JR. Exercise and well-being: a review of mental and physical health benefits associated with physical activity. Curr Opin Psychiatry 2005;18:189-93.

11. Teychenne M, Ball K, Salmon J. Sedentary behavior and depression among adults: a review. Int J Behav Med 2010;17:246-54.

12. Grisby $A B$, Anderson RJ, Freedland KE, et al. Prevalence of anxiety in adults with Diabetes: a systematic review. J Psychosom Res 2002;53:1053-60.

13. Cox DJ, Taylor AG, Singh $\mathrm{H}$, et al. Glycemic load, exercise, and monitoring blood glucose (GEM): a paradigm shift in the treatment of type 2 diabetes mellitus. Diabetes Res Clin Pract 2016;111:28-35.

14. Matthews CE, Chen KY, Freedson PS, et al. Amount of time spent in sedentary behaviors in the United States, 2003-2004. Am J Epidemiol 2008;167:875-81. 
15. Trost SG, Mclver KL, Pate RR. Conducting accelerometer-based activity assessments in field-based research. Med Sci Sports Exerc 2005;37:S531-43.

16. Matthew CE. Calibration of accelerometer output for adults. Med SCi Sports Exerc 2005;37:S512-22.

17. Balke B. A simple field test for the assessment of physical fitness. Rep Cuv Aeromed Res Inst US 1963;(53):1-8.

18. Ingle L. A review of the six-minute walk test: Its implication as a self-administered assessment tool. Eur J Cardiovasc Nurs 2009;8:232-4.

19. Faggiano P, D'Aloia A, Gualeni A, et al. Assessment of oxygen uptake during the 6-minute walking test in patients with heart failure: preliminary experience with a portable device. Am Heart $J$ 1997;134:203-6.

20. Guyatt GH, Thompson PJ, Berman LB, et al. How should we measure function in patients with chronic heart and lung disease? $J$ Chronic Dis 1985;38:517-24.

21. The World Health Organization Quality of Life (WHOQOL)-BREF. World Health Organization, 2004.

22. The WHOQOL Group. Development of the World Health Organization WHOQOL-BREF Quality of Life Assessment. Psychol Med 1998;28:551-8.

23. Anderson RM. Diabetes Empowerment Scale (DES). Ann Arbor, MI: University of Michigan, Diabetes Research \& Training Center, G1116-Towsley Center-0201, 48109-0201.

24. Anderson RM, Funnell MM, Fitzgerald JT, et al. The Diabetes Empowerment Scale: a measure of psychosocial self-efficacy. Diabetes Care. 2000;23:739-43.
25. Patient Health Questionnaire (PHQ-9), PHQ9 Copyright $\odot$ Pfizer Inc. A2662B 10-04-2005.

26. Kroenke K, Spitzer RL, Williams JB. The PHQ-9: validity of a brief depression severity measure. J Gen Intern Med 2001;16:606-13.

27. McGuir BE, Morrison TG, Hermanns N, et al. Short-form measures of diabetes-related emotional distress. The Problem Areas in Diabetes Scale (PAID)-5 and PAID-1. Diabetologia 2010;53:66-9.

28. Shuval K, Finley CE, Barlow CE, et al. Independent and joint effects of sedentary time and cardiorespiratory fitness on all-cause mortality: the Cooper Center Longitudinal Study. BMJ 2015;5:e008956.

29. Church TS, Blair SN, Cocrehan S, et al. Effects of aerobic and resistance training on hemoglobin A1c levels in patients with type 2 diabetes: a randomized controlled trial. JAMA 2010;304:2253-62.

30. Helmerhorst HJF, Wijndaele K, Brage S, et al. Objectively measured sedentary time may predict insulin resistance independent of moderate-and vigorous-intensity physical activity. Diabetes 2009;58:1776-9.

31. Ekelund U, Brage S, Griffin SJ, et al. Objectively measured moderate-and vigorous-intensity physical activity but not sedentary time predicts insulin resistance in high-risk individuals. Diabetes Care 2009;32:1081-6.

32. Carek PJ, Labstein SE, Carek SM. Exercise for the treatment of depression and anxiety.

33. Kennedy MM. Effect of exercise intensity on mood in step aerobics. J Sports Med Phys Fitness 1997;27:200-4.

34. Hamer M, Endrighi R, Poole L. Physical activity, stress reduction, and mood: insight into immunological mechanisms. Methods Miol Biol 2012;934:89-102. 